\title{
Normograma predictivo para evaluar la supervivencia en pacientes con metástasis vertebrales
}

\author{
Juan Gottlieb," Horacio Resano," Ignacio García," Claudio Bulos* \\ "Servicio de Ortopedia y Traumatología, Unidad de Columna, Hospital Sirio Libanés, ECICARO, Ciudad Autónoma \\ de Buenos Aires, Argentina \\ ${ }^{* *}$ Servicio de Ortopedia y Traumatología, Sanatorio Franchin, Ciudad Autónoma de Buenos Aires, Argentina
}

\begin{abstract}
RESUMEN
Introducción: La decisión respecto al procedimiento quirúrgico y la estrategia operatoria se basa en las expectativas de vida de los pacientes. Los sistemas difieren mucho en los parámetros evaluados y en el resultado del puntaje total. Por lo tanto, para un mismo paciente, se pueden calcular diferentes períodos de supervivencia y sugerir estrategias de tratamiento contradictorias. Objetivo: Analizar pacientes con metástasis vertebrales que fueron operados y evaluar la supervivencia mediante el normograma del SORG (Skeletal Oncology Research Group). Materiales y Métodos: Estudio retrospectivo que incluyó a 20 pacientes, de marzo de 2017 a marzo de 2019. Los criterios de inclusión fueron metástasis vertebrales con compresión medular o sin ella. Parámetros de evaluación: el normograma del SORG con probabilidad de supervivencia a los 30, 90 y 365 días, en la fase prequirúrgica, y la evaluación alejada: supervivencia a marzo de 2020. Resultados: 14 mujeres y 6 hombres, edad promedio: 67.9 años. Seguimiento de 6-36 meses. El puntaje promedio final fue de 222,4, lo que equivaldría a una supervivencia del $99 \%$ al mes, $78 \%$ a los 3 meses y $50 \%$ al año. Conclusión: En pacientes oncológicos con metástasis vertebrales, el normograma del SORG sería útil para predecir la supervivencia, sobre todo, al año.
\end{abstract}

Palabras clave: Metástasis vertebrales; supervivencia; cirugía espinal.

Nivel de Evidencia: IV

\section{Predictive Nomogram for Evaluating Survival in Patients with Spinal Metastases}

\begin{abstract}
Introduction: The decision of a surgical procedure and the surgical strategy are based on the life expectancies of the patients. The systems differ greatly in the parameters evaluated and in the result of the total score. Therefore, for the same patient, different survival periods can be calculated and contradictory treatment strategies suggested. Objective: To evaluate patients with spinal metastases who underwent surgery, evaluating survival according to the SORG (Skeletal Oncology Research Group) Nomogram. Materials and Methods: Retrospective study, March 2017 to March 2019. 20 patients. Inclusion criteria: spinal metastases, with or without spinal compression. Evaluation parameters: the SORG Nomogram with probability of survival, at 30, 90 and 365 days, in the preoperative phase and the remote evaluation: survival to March 2020. Results: 14 women and 6 men, average age: 67.9 years. Follow-up 6 to 36 months. Average final score: 222.4 points, which would be equivalent to a survival of $99 \%$ after a month, $78 \%$ after 3 months and $50 \%$ after a year. Conclusion: In cancer patients with spinal metastases, the SORG nomogram would be useful to predict survival, especially at one year.
\end{abstract}

Key words: Spinal metastases; survival; spine surgery.

Level of Evidence: IV

\section{INTRODUCCIÓN}

La enfermedad metastásica de la columna sigue siendo un gran desafío para los cirujanos espinales. Se debe elegir qué tipo de tratamiento utilizar para proporcionar el máximo control de la enfermedad (alivio del dolor, restablecimiento de la estabilidad y la función) con una mínima morbimortalidad operatoria.

Recibido el 2-7-2020. Aceptado luego de la evaluación el 21-9-2020 • Dr. JUAN GOTTLIEB • juangottlieb @ hotmail.com (ID https://orcid.org/0000-0001-8728-9801

Cómo citar este artículo: Gottlieb J, Resano H, García I, Bulos C. Normograma predictivo para evaluar la supervivencia en pacientes con metástasis vertebrales. Rev Asoc Argent Ortop Traumatol 2021;86(1):77-82. https://doi.org/10.15417/issn. 1852-7434.2021.86.1.1150 
La decisión respecto del procedimiento quirúrgico y la estrategia operatoria correspondiente, a menudo, se basa en las expectativas de vida estimadas de los pacientes.

Se han diseñado varios sistemas de evaluación para predecir la supervivencia y seleccionar la opción terapéutica ideal para cada paciente. Autores, como Tokuhashi, Tomita, Bauer, Ghori, por nombrar algunos, han propuesto tales sistemas.

El tipo de parámetros evaluados y el resultado del puntaje total difieren mucho entre los sistemas. Por lo tanto, para el mismo paciente, se pueden calcular diferentes períodos de supervivencia y sugerir estrategias de tratamiento contradictorias.

Como, en general, el tratamiento quirúrgico para las metástasis espinales es paliativo y estaría enfocado en mejorar la calidad de vida, es de suma importancia tener sistemas de pronóstico de supervivencia que sean reproducibles.

El objetivo de nuestro estudio fue evaluar a pacientes con metástasis vertebrales sometidos a cirugía valorando la supervivencia con el normograma prestablecido del SORG (Skeletal Oncology Research Group). Este normograma es un cuadro que incluye parámetros de pronóstico establecidos en una escala de puntos común, que oscilan de 0 a 100 para cada parámetro individual y luego se transpolan a una escala final de puntos que arroja la supervivencia aproximada, es decir, que acomodan variables continuas a un puntaje final y esta escala los traduce en probabilidades de supervivencia individuales.

\section{MATERIALES Y MÉTODOS}

Se llevó a cabo un estudio retrospectivo de pacientes con metástasis vertebrales operados entre marzo de 2017 y marzo de 2019. La muestra incluyó a 20 pacientes operados en nuestro centro. Los criterios de inclusión fueron: metástasis vertebrales, con compresión medular o sin ella. Se excluyó a los pacientes $<20$ años y aquellos con tratamiento oncológico no quirúrgico.

Dos cirujanos decidieron el tipo de tratamiento quirúrgico teniendo en cuenta el nivel de dolor, el grado de compresión medular y la inestabilidad mecánica.

La técnica quirúrgica utilizada fue la descompresión (si era necesaria) y la estabilización con tornillos pediculares por técnica percutánea, a cielo abierto o cifoplastia con balón.

Los parámetros de evaluación fueron los establecidos por el normograma del SORG (Figura 1) con la probabilidad de supervivencia, en la etapa prequirúrgica: hemoglobina, recuento de glóbulos blancos, edad, terapia sistémica previa, metástasis viscerales/cerebro, más de un segmento móvil con metástasis, tumor primario (tipo de tumor), escala del ECOG (Eastern Cooperative Oncology Group) (puntaje 3-4) y la supervivencia a los 30, 90 y 365 días; y en la evaluación alejada: supervivencia o no, constatada mediante un llamado telefónico en marzo de 2020.

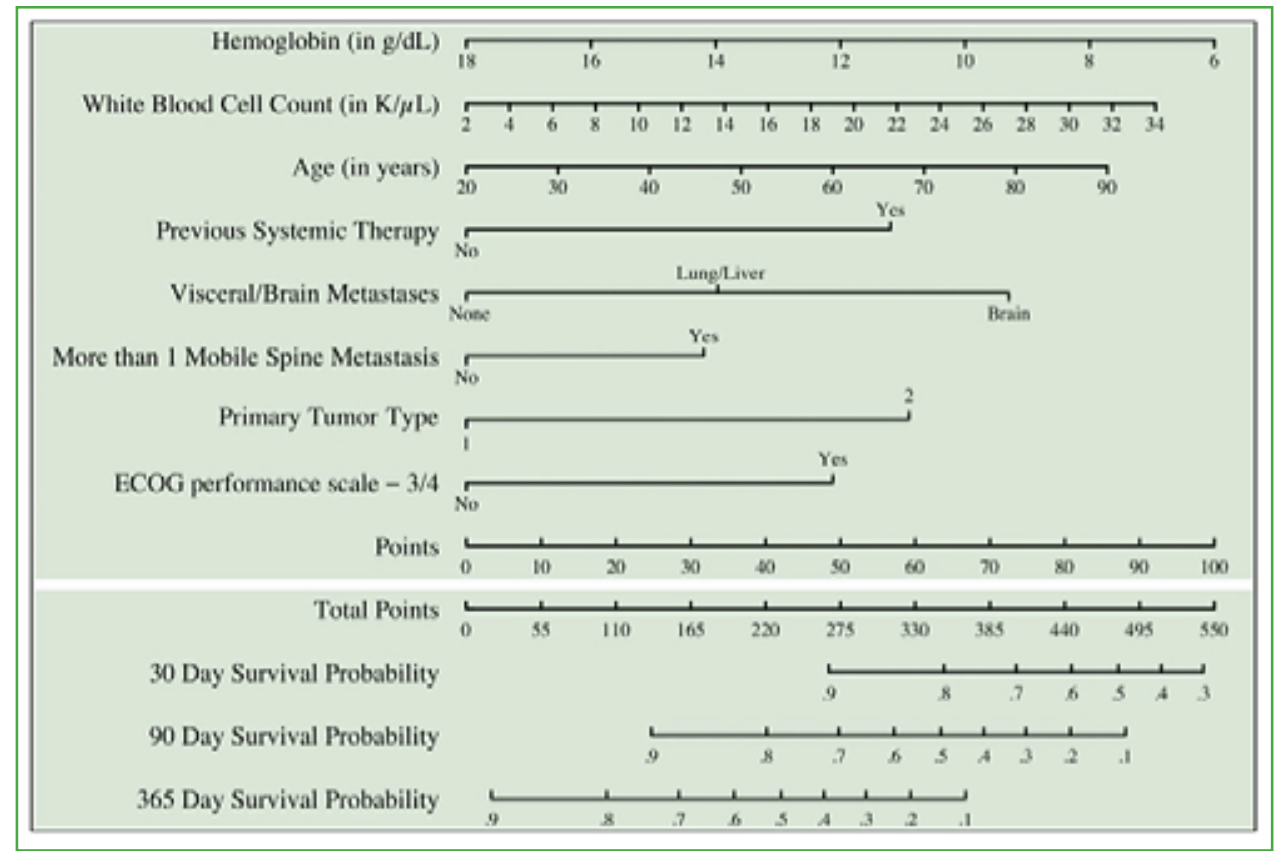

Figura 1. Normograma del SORG (Skeletal Oncology Research Group). 


\section{RESULTADOS}

Se incluyó a 14 mujeres y 6 hombres, con una edad promedio de 67.9 años (rango 21-90). El seguimiento mínimo fue de 6 meses y el máximo, de 36 meses calculado desde la fecha de la cirugía hasta la encuesta telefónica.

El tumor que más predominó fue el de mama (6 pacientes) seguido del mieloma múltiple y el linfoma (4 pacientes cada uno), de riñón y pulmón (2 pacientes, respectivamente) y, por último, de próstata e hígado (1 paciente cada uno). El sector más afectado fue el pasaje toracolumbar.

Las técnicas quirúrgicas empleadas fueron: cirugía a cielo abierto (8 casos), cifoplastia con balón (2 casos) y cirugía mínimamente invasiva (10 casos) (Tabla 1).

Tabla 1. Características de los pacientes

\begin{tabular}{|c|c|c|c|c|c|c|c|c|c|c|c|c|c|c|c|c|c|c|c|c|}
\hline & C.A. & G.M. & P.P. & W.M. & E.D. & G.M. & G.M. & P.E. & G.M. & B.M. & D.N. & D.C. & C.N. & G.M. & R.A. & F.E. & F.Z. & M.G. & V.I. & B.A \\
\hline Edad & 68 & 63 & 79 & 68 & 42 & 78 & 67 & 90 & 74 & 80 & 78 & 84 & 21 & 65 & 81 & 67 & 70 & 81 & 72 & 37 \\
\hline Sexo & M & $\mathrm{F}$ & M & $\mathrm{F}$ & $\mathrm{F}$ & $\mathrm{F}$ & $\mathrm{F}$ & $\mathrm{F}$ & $\mathrm{F}$ & F & M & M & M & $\mathrm{F}$ & $\mathrm{F}$ & $\mathrm{F}$ & $\mathrm{F}$ & M & $\mathrm{F}$ & $\mathrm{F}$ \\
\hline $\begin{array}{l}\text { Tipo de } \\
\text { tumor }\end{array}$ & Renal & Mama & Linfoma & Mama & $\begin{array}{l}\text { Mieloma } \\
\text { múltiple }\end{array}$ & Mama & Renal & Pulmón & Mama & $\begin{array}{l}\text { Mieloma } \\
\text { múltiple }\end{array}$ & $\begin{array}{c}\text { Mieloma } \\
\text { múltiple }\end{array}$ & Próstata & Linfoma & Mama & Mama & Pulmón & Linfoma & Linfoma & $\begin{array}{c}\text { Mieloma } \\
\text { múltiple }\end{array}$ & Hígado \\
\hline $\begin{array}{l}\text { Tipo de } \\
\text { cirugía }\end{array}$ & \multicolumn{9}{|c|}{ Mínimamente invasiva } & \multicolumn{9}{|c|}{ Cielo abierto } & \multicolumn{2}{|c|}{ Cifoplastia } \\
\hline
\end{tabular}

La hemoglobina promedio fue de 9,96 g/dl (rango 8-13) y el recuento de glóbulos blancos promedio, de 9370/ml (rango 5000-15.200). Solo cinco pacientes recibieron alguna terapia antes de la cirugía, relacionada con el tumor. Dos tuvieron metástasis en el cerebro y seis, metástasis en más de un nivel de la columna vertebral.

Diez pacientes tenían un puntaje 3-4 en la escala del ECOG; los restantes, un puntaje favorable de 1-2 (Figura 2).

El puntaje final promedio del normograma de toda la serie fue de 222,4 , lo que equivaldría a una supervivencia promedio del $99 \%$ al mes, $78 \%$ a los 3 meses y $50 \%$ al año.

\begin{tabular}{|c|c|c|}
\hline 0 & - & $\begin{array}{l}\text { Completamente activo, capaz de realizar toda actividad previa a la enfermedad sin } \\
\text { restricciones. }\end{array}$ \\
\hline 1 & - & $\begin{array}{l}\text { Restringido en la actividad física enérgica, pero está en régimen ambulatorio y es } \\
\text { capaz de realizar el trabajo de naturaleza ligera o sedentaria (como trabajo de la } \\
\text { casa ligero o trabajo de oficina). }\end{array}$ \\
\hline 2 & - & $\begin{array}{l}\text { En régimen ambulatorio y capaz de cuidar de sí mismo, pero incapaz de realizar } \\
\text { ninguna actividad de trabajo. Tiene que quedarse en cama durante menos del } 50 \% \\
\text { de las horas de vigilia. }\end{array}$ \\
\hline 3 & - & $\begin{array}{l}\text { Capacidad limitada de cuidar de sí mismo, confinado a la cama o una silla durante } \\
\text { más del } 50 \% \text { de las horas de vigilia. }\end{array}$ \\
\hline 4 & - & $\begin{array}{l}\text { Totalmente incapaz. No puede cuidar de sí mismo. Totalmente confinado a la cama } \\
\text { o una silla. }\end{array}$ \\
\hline
\end{tabular}

Figura 2. Escala del estado funcional del ECOG (Eastern Cooperative Oncology Group). 
Hasta marzo de 2020, según la encuesta telefónica, seis pacientes (30\%) habían muerto por complicaciones sistémicas del tumor y dos (10\%), por causas no relacionadas con su enfermedad de base (paro cardiorrespiratorio, uno a los 5 días de la cirugía y el otro, a los 2 meses, ya constatado en el seguimiento sin necesidad de hacer el llamado telefónico). Los 12 pacientes (60\%) restantes seguían vivos.

En cinco $(25 \%)$ de los seis pacientes que murieron a causa del tumor, el óbito se produjo entre los 6 meses y el año de la cirugía, salvo uno con mieloma múltiple que sobrevivió dos años. Todos ellos tenían un puntaje del normograma del SORG $>280$ (rango 285-320) que se traduce en una probabilidad de supervivencia de alrededor del $25 \%$ al año.

En los 12 pacientes vivos, el puntaje del normograma del SORG promedio fue de 190,4 (rango 110-310), lo que sería una supervivencia estimada superior al $55 \%$ en un año.

El tiempo promedio que llevan vivos desde la cirugía hasta la encuesta telefónica es de 22.2 meses (rango 12-36) (Tabla 2).

Tabla 2. Parámetros pre y posquirúrgicos evaluados

\begin{tabular}{|c|c|c|c|c|c|c|c|c|c|c|c|c|c|c|c|c|c|c|c|c|}
\hline & F.Z. & B.M. & D.N. & E.D. & D.C. & G.M. & C.N. & F.E. & G.M. & M.G. & G.M. & P.E. & C.A. & R.A. & P.P. & W.M. & G.M. & V.I. & G.M. & B.A \\
\hline $\begin{array}{l}\text { Hemoglo- } \\
\text { bina }\end{array}$ & 9 & 8 & 11 & 10 & 11 & 9 & 9 & 10 & 9 & 9 & 10 & 9 & 11,1 & 13 & 9.8 & 11,4 & 9 & 9 & 11,9 & 10 \\
\hline $\begin{array}{l}\text { Glóbulos } \\
\text { blancos }\end{array}$ & 8300 & 15.200 & 10.500 & 7000 & 8100 & 9000 & 14.000 & 8000 & 7300 & 5000 & 8000 & 8300 & 7200 & 15.000 & 7300 & 7400 & 7500 & 11.000 & 15.200 & 8000 \\
\hline Edad & 70 & 80 & 78 & 42 & 84 & 78 & 21 & 67 & 67 & 81 & 63 & 90 & 68 & 81 & 79 & 68 & 74 & 72 & 65 & 37 \\
\hline $\begin{array}{l}\text { Terapia } \\
\text { previa }\end{array}$ & No & No & $\mathrm{N}_{0}$ & No & $\mathrm{N}_{0}$ & No & Sí & No & No & No & No & Sí & No & No & No & Sí & Sí & No & No & Sí \\
\hline $\begin{array}{l}\text { Metástasis } \\
\text { en órganos }\end{array}$ & No & No & No & No & No & No & No & Cerebro & No & No & No & No & No & No & Cerebro & No & No & No & No & No \\
\hline $\begin{array}{l}\text { Más de una } \\
\text { metástasis }\end{array}$ & No & Sí & No & No & No & No & No & No & No & Sí & No & No & No & Sí & No & No & Sí & No & Sí & Sí \\
\hline $\begin{array}{l}\text { Tumor } \\
\text { primario }\end{array}$ & Linfoma & $\begin{array}{l}\text { Mieloma } \\
\text { múltiple }\end{array}$ & $\begin{array}{l}\text { Mieloma } \\
\text { múltiple }\end{array}$ & $\begin{array}{l}\text { Mieloma } \\
\text { múltiple }\end{array}$ & Próstata & Mama & Linfoma & Pulmón & Renal & Linfoma & Mama & Pulmón & Renal & Mama & Linfoma & Mama & Mama & $\begin{array}{l}\text { Mieloma } \\
\text { múltiple }\end{array}$ & Mama & Hígado \\
\hline ECOG & 1 & 2 & 1 & 1 & 1 & 1 & 2 & 2 & 1 & 1 & 2 & 2 & 2 & 2 & 2 & 1 & 1 & 1 & 2 & 2 \\
\hline Puntaje & 155 & 285 & 155 & 110 & 160 & 170 & 290 & 320 & 195 & 183 & 190 & 300 & 185 & 235 & 285 & 190 & 305 & 195 & 230 & 310 \\
\hline $\begin{array}{l}\text { Supervi- } \\
\text { vencia } \\
\text { a } 1 \text { mes }\end{array}$ & $99 \%$ & $85 \%$ & $99 \%$ & $99 \%$ & $99 \%$ & $99 \%$ & $85 \%$ & $85 \%$ & $99 \%$ & $99 \%$ & $99 \%$ & $85 \%$ & $99 \%$ & $99 \%$ & $85 \%$ & $99 \%$ & $85 \%$ & $99 \%$ & $99 \%$ & $85 \%$ \\
\hline $\begin{array}{l}\text { Supervi- } \\
\text { vencia a } 3 \\
\text { meses }\end{array}$ & $85 \%$ & $60 \%$ & $85 \%$ & $90 \%$ & $85 \%$ & $85 \%$ & $60 \%$ & $60 \%$ & $80 \%$ & $85 \%$ & $85 \%$ & $60 \%$ & $85 \%$ & $75 \%$ & $65 \%$ & $85 \%$ & $65 \%$ & $85 \%$ & $75 \%$ & $60 \%$ \\
\hline $\begin{array}{l}\text { Supervi- } \\
\text { vencia } \\
\text { al año }\end{array}$ & $70 \%$ & $25 \%$ & $70 \%$ & $80 \%$ & $65 \%$ & $60 \%$ & $25 \%$ & $25 \%$ & $60 \%$ & $60 \%$ & $60 \%$ & $25 \%$ & $60 \%$ & $45 \%$ & $20 \%$ & $60 \%$ & $30 \%$ & $60 \%$ & $45 \%$ & $25 \%$ \\
\hline $\begin{array}{l}\text { Fecha de } \\
\text { la cirugía }\end{array}$ & $\begin{array}{c}\text { Marzo } \\
2017\end{array}$ & $\begin{array}{l}\text { Mayo } \\
2017\end{array}$ & $\begin{array}{l}\text { Junio } \\
2017\end{array}$ & $\begin{array}{l}\text { Julio } \\
2017\end{array}$ & $\begin{array}{l}\text { Julio } \\
2017\end{array}$ & $\begin{array}{c}\text { Sep- } \\
\text { tiembre } \\
2017\end{array}$ & $\begin{array}{l}\text { Diciem- } \\
\text { bre } 2017\end{array}$ & $\begin{array}{c}\text { Enero } \\
2018\end{array}$ & $\begin{array}{c}\text { Marzo } \\
2018\end{array}$ & $\begin{array}{l}\text { Abril } \\
2018\end{array}$ & $\begin{array}{l}\text { Mayo } \\
2018\end{array}$ & $\begin{array}{c}\text { Agosto } \\
2018\end{array}$ & $\begin{array}{c}\text { Sep- } \\
\text { tiembre } \\
2018\end{array}$ & $\begin{array}{c}\text { Octubre } \\
2018\end{array}$ & $\begin{array}{l}\text { Diciem- } \\
\text { bre } 2018\end{array}$ & $\begin{array}{c}\text { Febrero } \\
2019\end{array}$ & $\begin{array}{c}\text { Febrero } \\
2019\end{array}$ & $\begin{array}{c}\text { Marzo } \\
2019\end{array}$ & $\begin{array}{c}\text { Marzo } \\
2019\end{array}$ & $\begin{array}{c}\text { Marzo } \\
2019\end{array}$ \\
\hline $\begin{array}{l}\text { Encuesta } \\
\text { telefónica }\end{array}$ & Vive & Óbito & Vive & Vive & Vive & Vive & Óbito & Óbito & Óbito & Óbito & Vive & Óbito & Vive & Vive & Óbito & Vive & Óbito & Vive & Vive & Vive \\
\hline $\begin{array}{l}\text { Seguimien- } \\
\text { to posope- } \\
\text { ratorio }\end{array}$ & $\begin{array}{c}36 \\
\text { meses }\end{array}$ & 24 meses & 31 meses & 32 meses & $\begin{array}{c}32 \\
\text { meses }\end{array}$ & $\begin{array}{c}30 \\
\text { meses }\end{array}$ & 9 meses & 6 meses & $\begin{array}{c}5 \text { días } \\
\text { (paro } \\
\text { cardio- } \\
\text { rrespi- } \\
\text { rato- } \\
\text { rio) }\end{array}$ & $\begin{array}{c}2 \text { meses } \\
\text { (paro } \\
\text { cardio- } \\
\text { rrespira- } \\
\text { torio) }\end{array}$ & $\begin{array}{c}22 \\
\text { meses }\end{array}$ & $\begin{array}{c}12 \\
\text { meses }\end{array}$ & $\begin{array}{c}18 \\
\text { meses }\end{array}$ & $\begin{array}{c}17 \\
\text { meses }\end{array}$ & 6 meses & $\begin{array}{c}13 \\
\text { meses }\end{array}$ & $\begin{array}{c}13 \\
\text { meses }\end{array}$ & 12 meses & $\begin{array}{c}12 \\
\text { meses }\end{array}$ & $\begin{array}{c}12 \\
\text { meses }\end{array}$ \\
\hline
\end{tabular}




\section{DISCUSIÓN}

El tratamiento actual de las metástasis vertebrales está enfocado en prolongar la expectativa de vida y mejorar la calidad de vida. Cuando el cirujano elige qué tipo de tratamiento realizará, es esencial que evalúe si el paciente lo tolerará teniendo en cuenta el estado actual y su probable supervivencia. Por lo general, esta estimación de supervivencia se basa en la experiencia del cirujano más que en algún algoritmo preestablecido.

La importancia de obtener esta información ayuda a establecer expectativas apropiadas para el paciente, la familia y el médico. Los datos sobre el costo, el riesgo y la calidad de vida no siempre son correctos, pero si estos datos se pudieran obtener adecuadamente, se reduciría el riesgo y serviría para definir el tratamiento más adecuado para cada paciente.

Numerosos estudios identificaron factores de riesgo y crearon sistemas predictvios para evaluar la supervivencia. En 1990, Tokuhashi y cols. ${ }^{1}$ crearon el primer algoritmo basado en 64 pacientes operados por enfermedad metastásica de la columna. Eligieron seis parámetros para el algoritmo de supervivencia en función de su experiencia con pacientes tratados con cirugía. Este puntaje oscilaba entre 0 y 12. Estos autores sugirieron la cirugía de escisión para pacientes con $>9$ puntos y métodos quirúrgicos paliativos para aquellos con $<5$ puntos. En 2005 , lo modificaron asignando más importancia a cánceres primarios específicos. ${ }^{2}$ Este puntaje es uno de los más utilizados en la actualidad. En 1995, Bauer y cols. ${ }^{3}$ reportaron otro sistema de evaluación de la supervivencia con 241 pacientes que sufrían metástasis espinales y en las extremidades. Asignaron 1 punto por cada parámetro elegido y categorizaron a los pacientes en tres grupos; cada grupo correspondía a una estrategia quirúrgica sugerida (sin cirugía, cirugía posterior y cirugía combinada).

En 2001, Tomita y cols. ${ }^{4}$ presentaron un algoritmo de supervivencia como guía para decidir el tipo de tratamiento en 67 pacientes con metástasis en la columna. Con el puntaje total obtenido, se clasificó a los pacientes en cuatro grupos, cada uno correspondiente a una estrategia quirúrgica sugerida (escisión amplia o marginal, escisión marginal o intralesional, cirugía paliativa y tratamiento no quirúrgico u oncológico, solo de apoyo).

En 2015, Ghori y cols. ${ }^{5}$ estudiaron la supervivencia al año de la cirugía en 307 pacientes con enfermedad metastásica de la columna. Su algoritmo de supervivencia abarcó dos factores de riesgo recién identificados en pacientes con enfermedad metastásica de la columna vertebral (estado ambulatorio y albúmina sérica).

Todos estos sistemas de predicción, muchas veces, arrojan resultados que pueden diferir en un mismo paciente y hacen elegir estrategias de tratamiento erróneas. ${ }^{6}$

Un sistema de puntaje eficaz reduce el riesgo de administrar un tratamiento insuficiente a los pacientes con supervivencia más prolongada o un tratamiento excesivo a aquellos con un mal pronóstico. ${ }^{7}$ La cirugía radical puede ser estresante, a veces, generar complicaciones posoperatorias y puede causar un retraso de la terapia sistémica adyuvante, que no sería lo ideal debido a la mejora que estas terapias logran actualmente para controlar el crecimiento tumoral. Como alternativa, la cirugía mínimamente invasiva indicada a un paciente con una supervivencia a largo plazo puede no ser suficiente para asegurar una buena calidad de vida durante un período prolongado.

En 2016, el SORG ${ }^{8}$ ideó un normograma agregando algunos factores de riesgo a los ya propuestos, como la hemoglobina , el recuento de glóbulos blancos y el estado de salud del paciente según el ECOG (Figura 1), y logró una muy buena validación, sobre todo en cuanto a la supervivencia a los 30, 90 y 365 días.

En nuestra serie de casos, según este puntaje, los pacientes con puntajes más altos (>285) tenían una expectativa de vida estimada promedio al año $<25 \%$, esto coincide con los cinco pacientes $(25 \%)$ que murieron dentro del año de la cirugía. Asimismo, en los pacientes que seguían vivos, según los estimativos del normograma, el puntaje de supervivencia teórica promedio fue $>60 \%$ (190 puntos) al año y, si lo comparamos con el resultado real de nuestra muestra, 12 pacientes (60\%) vivos a la fecha de corte, podemos decir que hay coincidencia entre ambos.

Este estudio tiene tres grandes limitaciones: 1) la poca casuística $(\mathrm{n}=20), 2)$ la diversidad de las metástasis y 3) las metástasis tratadas con tres técnicas quirúrgicas diferentes: estabilización percutánea, a cielo abierto o cifoplastia. Por lo tanto, creemos que es necesario llevar a cabo estudios multicéntricos con casuísticas más grandes y, a su vez, agrupar los diferentes tipos de metástasis y técnicas quirúrgicas utilizadas, para lograr una más exacta validación de este nuevo tipo de puntaje pronóstico.

\section{CONCLUSIÓN}

En pacientes oncológicos con metástasis vertebrales, el normograma del SORG sería útil para predecir la supervivencia, sobre todo al año, y ayudar a la planificación preoperatoria. 
Conflicto de intereses: Los autores no declaran conflictos de intereses.

ORCID de H. Resano: https://orcid.org/0000-0002-2772-803X

ORCID de I. García: https://orcid.org/0000-0002-8837-8520

ORCID de C. Bulos: https://orcid.org/0000-0001-6925-2722

\section{BIBLIOGRAFÍA}

1. Tokuhashi Y, Matsuzaki H, Toriyama S, Kawano H, Ohsaka S. Scoring system for the preoperative evaluation of metastatic spine tumor prognosis. Spine (Phila Pa 1976) 1990;15(11):1110-3.

https://doi.org/10.1097/00007632-199011010-00005

2. Tokuhashi Y, Matsuzaki H, Oda H, Oshima M, Ryu J. A revised scoring system for preoperative evaluation of metastatic spine tumor prognosis. Spine (Phila Pa 1976) 2005;30(19):2186-91.

https://doi.org/10.1097/01.brs.0000180401.06919.a5

3. Bauer HC, Wedin R. Survival after surgery for spinal and extremity metastases. Prognostication in 241 patients. Acta Orthop Scand 1995;66(2):143-6. https://doi.org/10.3109/17453679508995508

4. Tomita K, Kawahara N, Kobayashi T, Yoshida A, Murakami H, Akamaru T. Surgical strategy for spinal metastases. Spine (Phila Pa 1976) 2001;26(3):298-306. https://doi.org/10.1097/00007632-200102010-00016

5. Ghori AK, Leonard DA, Schoenfeld AJ. Modeling 1-year survival after surgery on the metastatic spine. Spine J 2015;15(11):2345-50. https://doi.org/10.1016/j.spinee.2015.06.061

6. Leithner A, Radl R, Gruber G, Windhager R. Predictive value of seven preoperative prognostic scoring systems for spinal metastases. Eur Spine J 2008;17(11):1488-95. https://doi.org/10.1007/s00586-008-0763-1

7. Zoccali C, Skoch J, Borgstrom M, Baaj A. The Tokuhashi score: effectiveness and pitfalls. Eur Spine J 2016;25(3):673-8. https://doi.org/10.1007/s00586-015-4339-6

8. Paulino Pereira NR, Mclaughlin L, Janssen SJ, van Dijk CN, Bramer JAM, Laufer I, et al. The SORG nomogram accurately predicts 3- and 12-months survival for operable spine metastatic disease: External validation. J Surg Oncol 2017;115(8):1019-27. https://doi.org/10.1002/jso.24620 